\title{
Automatic Monitoring of Bridges using Electronic Inclinometers
}

\author{
Olivier BURDET \\ Lecturer \\ EPFL \\ Lausanne, Switzerland \\ Olivier Burdet, born 1960, is a \\ lecturer in reinforced and \\ prestressed concrete. His \\ research interests include \\ serviceability, bridge design \\ and monitoring techniques. \\ $\mathrm{He}$ is a member of WC 3 and \\ of the Publication's Committee \\ of IABSE SEI Journal.
}

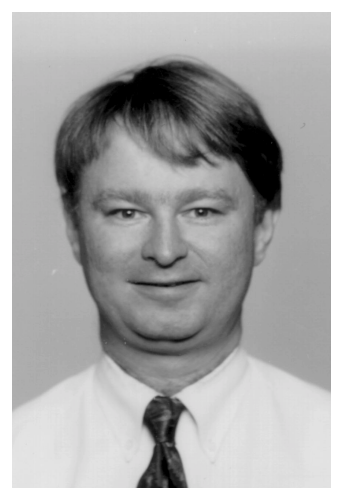

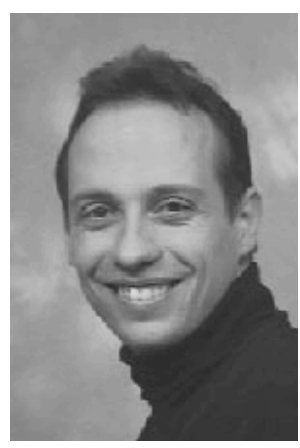

\section{Summary}

The paper presents a new method for the automatic monitoring of bridge deflections. Based on the use of electronic high precision inclinometers, this method permits to accurately reconstruct the deflected shape of the bridge and to follow its long-term evolution. A practical and efficient method for the reconstruction of the deflected shape from measured rotations is proposed. The example of an actual bridge in Western Switzerland, in which the system is operational, is described. The results obtained correspond well with those obtained from independent measurements.

Keywords: prestressed concrete, in situ measurements, bridge monitoring, long-term deflections, intelligent structures, bridge management

\section{Introduction}

Automatic monitoring is an efficient way to preserve existing structures, by following their behavior in service. The data gathered in the monitoring can be used for the evaluation of the condition of the bridge. The method presented in the paper allows to automatically and accurately follow the deflections of all kinds of structures. Based on the measurement of rotations, the complete deflected shape of a bridge can be reconstructed using pre-calculated deflected shapes. This method has been successfully implemented for the long-term monitoring of the Mentue bridge in Western Switzerland. The method can also be used for short-term measurements, for example during a loading test.

\section{Automatic monitoring}

Monitoring is rapidly becoming an usual manner to follow the behavior in service of existing structures. Besides giving a more accurate picture of the effective behavior in service, which is very important for new of particularly complex structures, automatic monitoring allows to follow the evolution over time of the measured performance of a structure. Regular checks of the present performance compared to initial values give a more precise idea of the aging process and possible deterioration of the structure than simple visual inspections can.

In the context of modern bridge management systems, automatic continuous monitoring of structures is an effective way to gather quantitative information to serve as input into the evaluation module for predictions and planning of repair works. As a complement to the more traditional methods of observation, automatic monitoring permits a finer analysis. Finally, it is clear that automatic methods have an advantage in a world where equipment becomes increasingly cheaper, while cost of labor is high and availability of skilled personal is low.

The method proposed here is innovative in its use of high precision electronic inclinometers for the monitoring of box girder bridges. Its simplicity of installation and operation, combined with its high accuracy are crucial advantages for the systematic surveillance of civil engineering structures. The 
algorithm proposed for the reconstruction of the deflected shape is particularly robust and well suited for the monitoring of all sorts of bridges, and potentially of other types of structures. These advantages should lead to a widespread application of this method.

\section{Measurement methodology}

The methodology used for the automatic deflection monitoring consists in the use of a network of small high accuracy electronic inclinometers connected to a centrally located PC running the data acquisition program. All the equipment can easily be installed in the box girder of a bridge. The sensors, with a resolution of $1 \mu \mathrm{rad}$ (one millimeter per kilometer) are simply mounted at the surface of the concrete, either on simple mounts as shown in figure 1, or on more sophisticated devices (fig. 2) that permit a recalibration by a rotation of $180^{\circ}$ around a vertical axis. The sensors are regularly activated to take measurements over a specified period of time. At the end of that period (typically 10 seconds), all instruments report the measured values, either in the form of an average over the measurement period, or as individual values. The values are then stored locally and displayed on the computer screen. Simultaneous temperature measurements of relevant positions in the bridge cross section are typically also taken, to ensure a proper interpretation of the measurements.

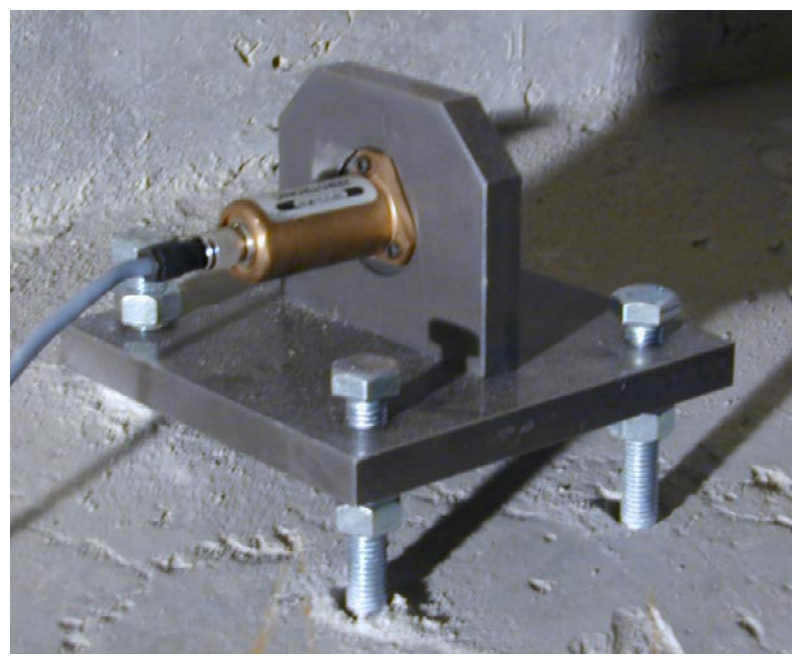

Fig. 1 Simple support for a fixed inclinometer
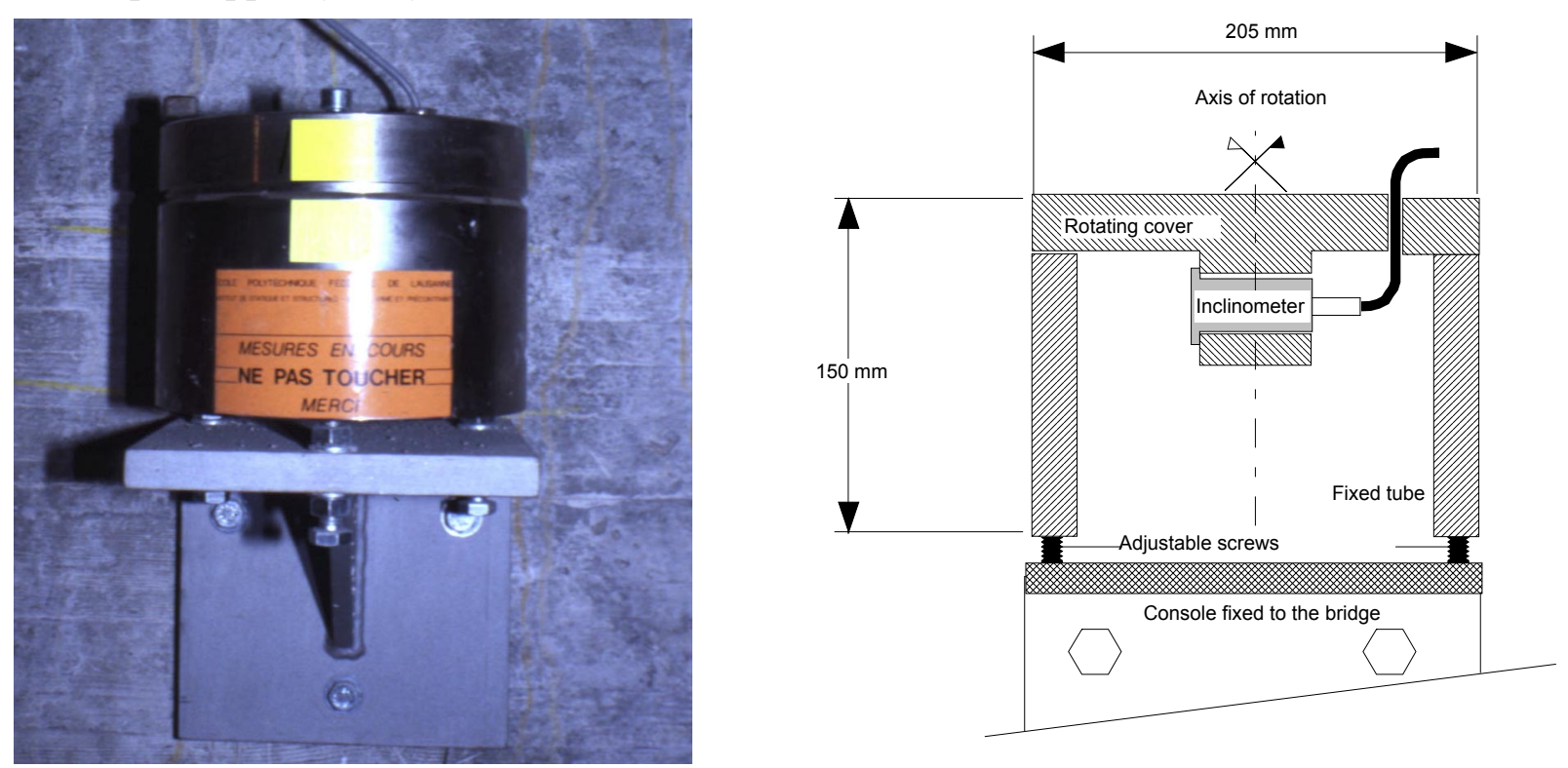

a) Photo of the device

b) Schematic and main dimensions

Fig. 2 Rotation system for the correction of drifting over time 
The sensors themselves have a sampling frequency of 10 to $20 \mathrm{~Hz}$ depending on the model, but the bandwidth of the sensor network and practical limitations restrict the actual frequency of measurements in situ. Measurements are typically taken in bursts of 20 to 100 with a frequency of $10 \mathrm{~Hz}$ and then all values are reported to the computer. The standard deviation of the series of measurements gives a good idea of the condition of the bridge during the measurement. It is thus easy to automatically filter out values with a high standard deviation, that indicate the presence of traffic on the structure during the measurements. To allow the effective collection of data even in periods of dense traffic a measurement period of one per minute is typically used. This ensures that at least some measurements will be valid for each hour.

\section{Reconstruction of the deflected shape}

Although rotations can give valid indications on the condition of a structure, it is generally necessary to reconstruct the deflected shape of the structure from the measured rotations to permit an easier interpretation. Once rotation values have been acquired, their values are combined to reconstruct the corresponding deflected shape. The simplest approach consists of a simple polynomial reconstruction. Three inclinometers define a second order polynomial. By integrating once the polynomial, and setting the initial deflection to zero $y(0)=0$, the deflected shape is obtained. Figure 3 illustrates this process for three inclinometers located at $x=0, x=0.3 \ell$ and $x=\ell$. As can be seen from fig. $3 a$, this method gives good results for the deflection at mid-span. It is however noticeable that the curve $(N=3)$ does not come back to zero at the right support. This effect disappears if more inclinometers are used, or if the polynomial is defined to pass through zero at the right support (fig. $3 b, N=4$ ). This has, however, the undesirable effect that potential support settlements cannot be observed. In general, if only the mid-span deflection is desired, this method yields acceptable results.

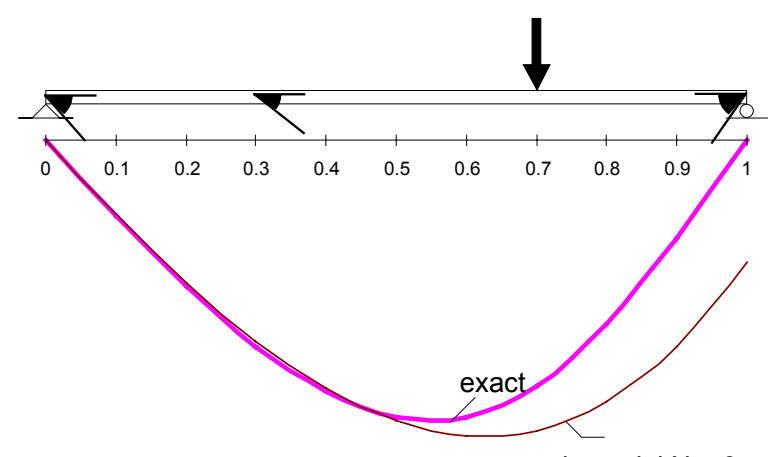

polynomial $\mathrm{N}=3$

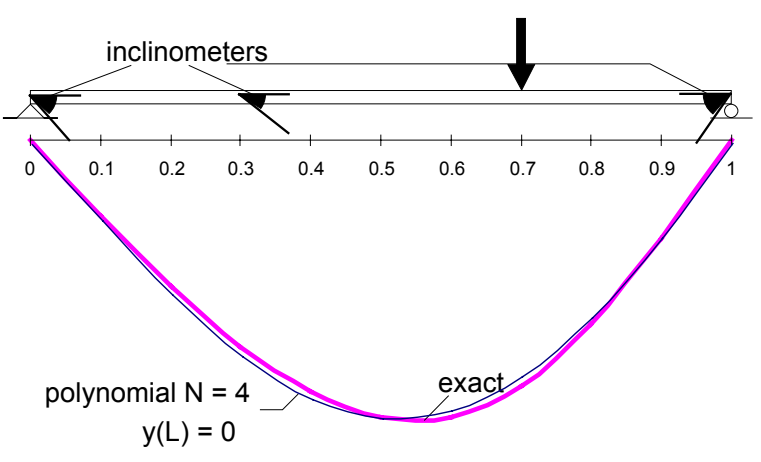

a) polynomial through $y(0)=0$

b) polynomial through $y(0)=0$ and $y(\ell)=0$

Fig. 3 Reconstruction of the deflected shape by simple polynomials

While reconstruction of the deflected shape by simple polynomials has been found to be somewhat effective [5], it has been found that its practical application is rather difficult, and that it is quite sensitive to measurement errors, most notably because it forces the polynomial to pass exactly through all measured values. As a consequence, a pathological behavior with a lot of zeroes between supports has been occasionally observed (fig. 11).

As a consequence, a different approach was used. In this method, the deflected shapes are reconstructed by means of linear combinations of a series of pre-calculated deflected shapes. The basic idea is that structures very generally deflect following smooth " engineer's curves ", obtained from actual deflection calculations under canonical load cases (fig. 4). A linear combination of a certain number of these curves, yields a curve that is also a smooth curve. Essentially, each deflected shape respects the bridge properties, and can account for discontinuities, such as sudden changes of inertia, much better than polynomials can. 


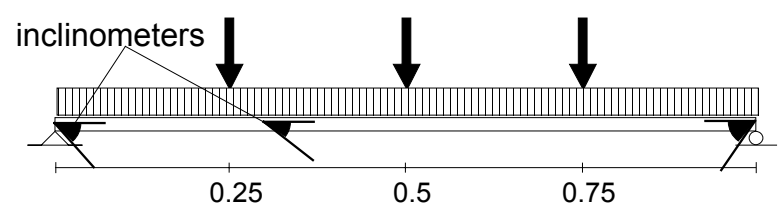

Fig. 4 Load cases used for the pre-calculation of the base shapes

Figure 5 shows that for the same configurations as in figure 3 the results are very close to the actual deflected shape. The results from the reconstruction by pre-calculated deflected shapes is very comparable with the results of the polynomial with no displacement at both supports shown previously in figure $3 b$. An interesting feature of this method is that the reconstructed deflected shape yields additional information on the way the best deflected shape is obtained. In this case, where all combinations of two of the four loading patterns of fig. 4 are used, the combination that gives the best result (combined rotation vector closest on the sense of least squares to the measured rotation vector) is $10 \%$ of uniform loading plus $102 \%$ of unit load at $0.75 \ell$. The interpretation of these coefficients, and their variation over time should give additional information about the actual behavior of the bridge. It is for example expected that significant changes in the structure will lead to significantly different factors used in the controlling combination, thus giving a signal that the structure has changed.

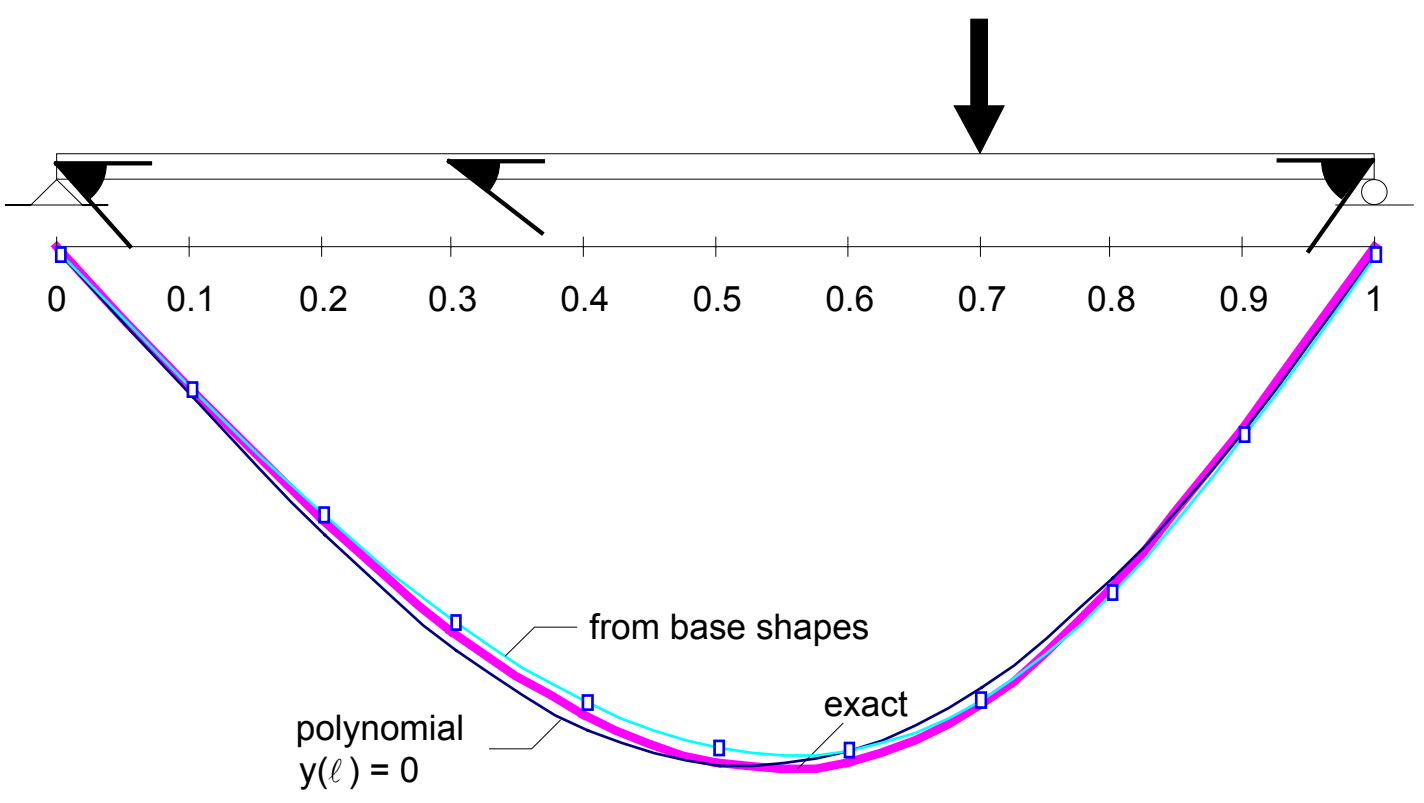

Fig. 5 Reconstruction of the deflected shape by combinations of pre-calculated deflected shapes

\section{Pilot Project : the Mentue Bridges}

The Mentue bridges are twin bridges that carry the future A1 motorway from Lausanne to Bern. Each bridge, similar in design, has an overall length of approximately $565 \mathrm{~m}$, and a width of $13.46 \mathrm{~m}$, designed to carry two lanes of traffic and an emergency lane. The bridges cross a deep valley with steep sides (fig. 6). The balanced cantilever design results from a bridge competition. The very high concrete piers were built using climbing formwork, after which the construction of the balanced cantilever started (fig. 7). 


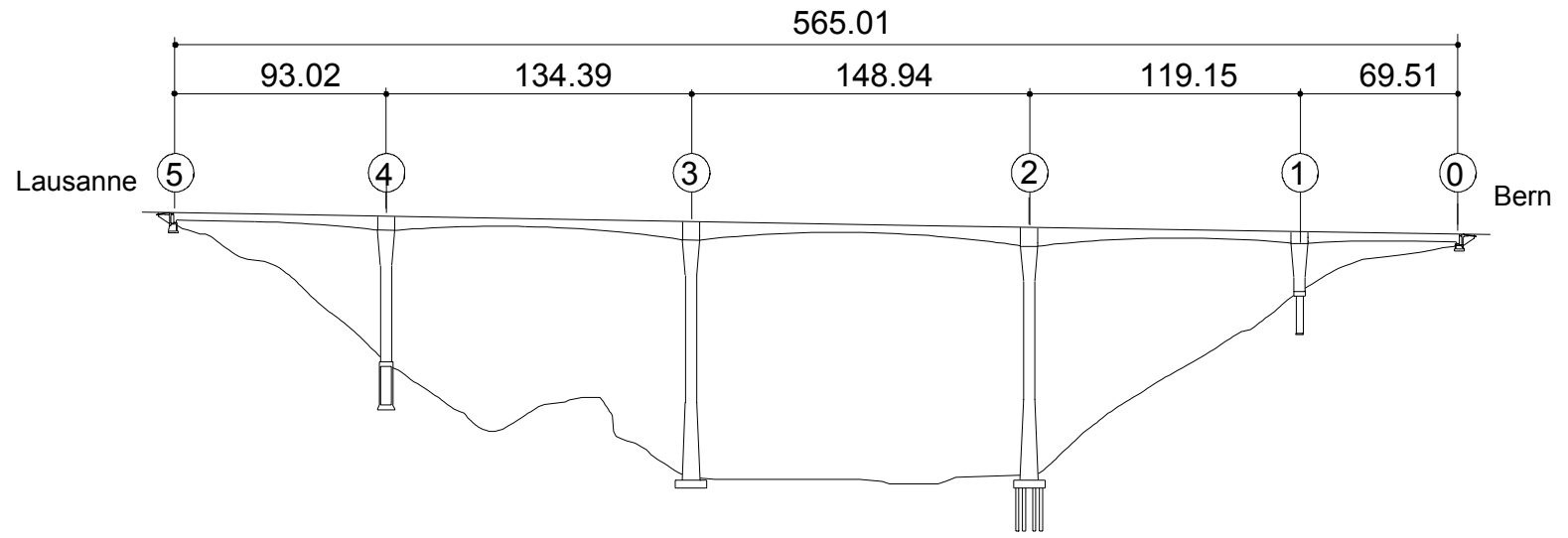

Fig. 6 Elevation of the Mentue bridges

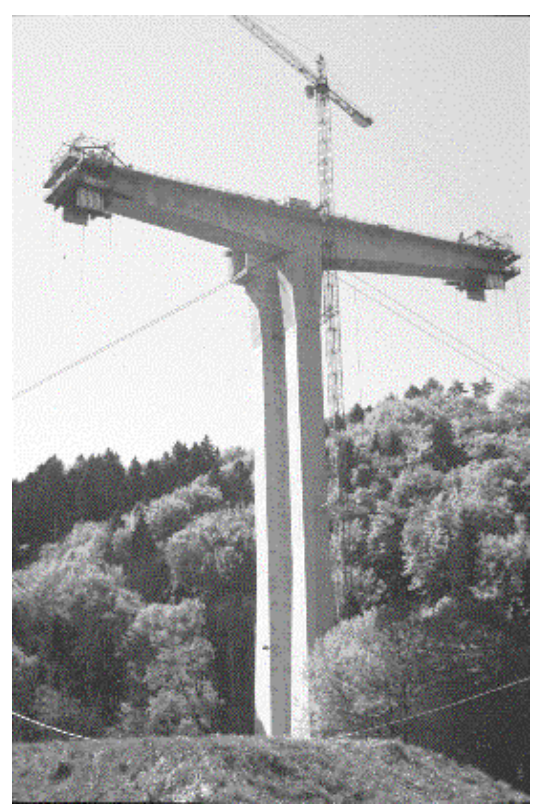

Fig. 7 Mentue bridges during the free cantilevering

Because bridges built by the balanced cantilevering method have been prone to exhibit larger than anticipated deflections in the past $[3,6]$, a pilot project was initiated to set up and operate a network of inclinometers in the main span of the North Mentue bridge. Five inclinometers are located in the main span as shown in figure 8. Additional inclinometers have also been placed at both ends of the bridge to monitor the movement of expansion joints. A complete network of hydrostatic leveling devices has also been installed in each bridge, allowing for a reliable comparison of measurements $[2,3,4]$.

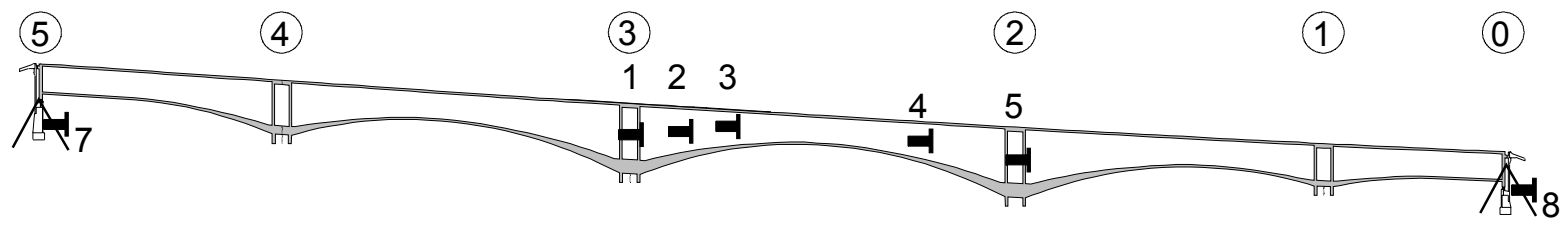

Fig. 8 Location of the inclinometers in the main span of the North bridge 


\section{Results from the measurements}

The system has been operational since more than two years. After some initial problems, mainly related to the required reliability of the controlling computer for operation under severe weather conditions, the system is functioning properly. So far, no significant long term deflection has been observed. Daily and seasonal temperature are the main effects on the structure, which is not yet open to traffic (fig. 9).

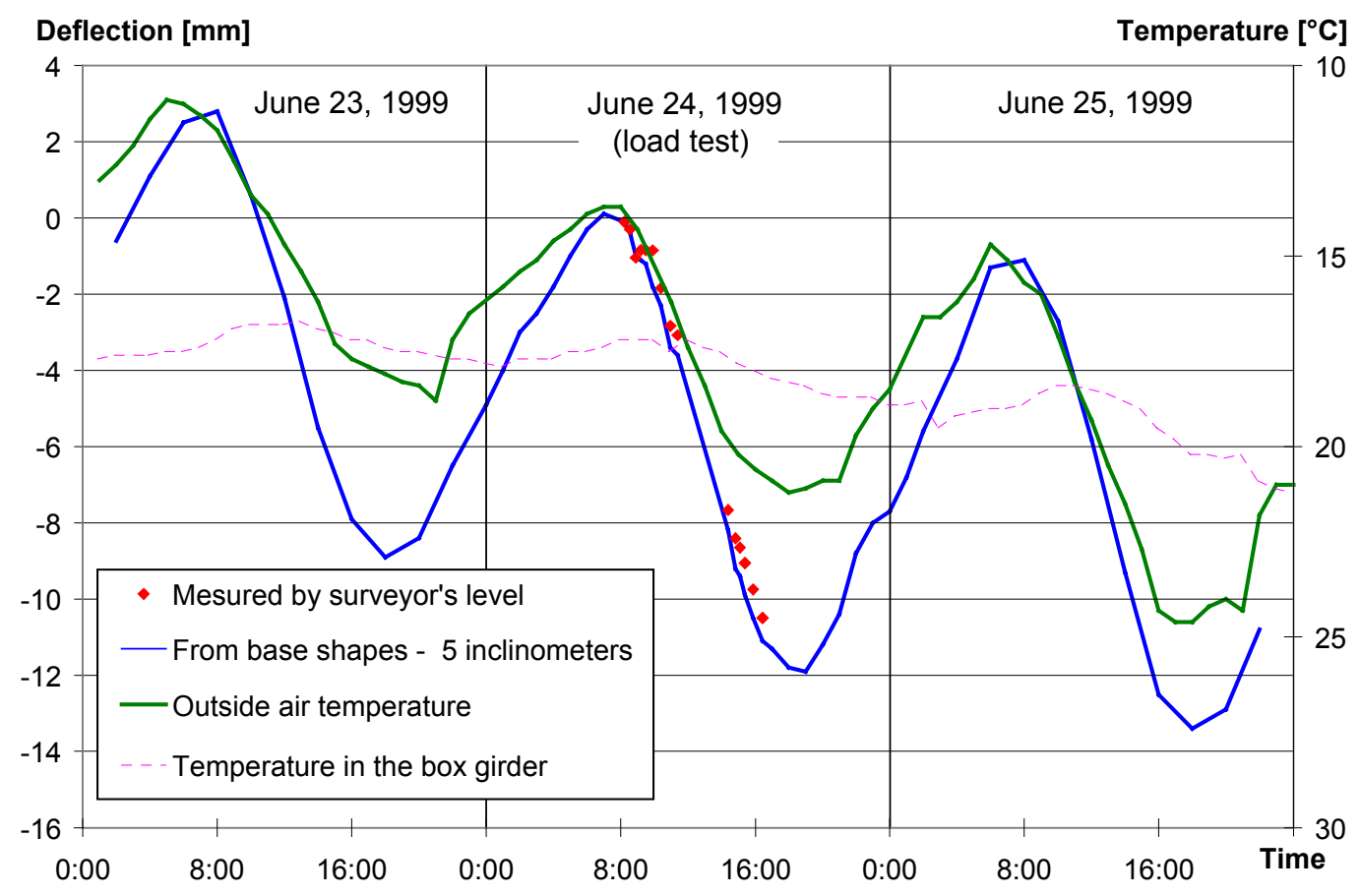

Fig. 9 Mid-span deflection and temperature over three days

One opportunity to test the measuring system was offered during the static load test of the bridge. The system of inclinometers was active during that test, which allows a direct comparison of deflections reconstructed from rotations and direct measurements. Figure 10 shows the measured and reconstructed deflected shapes for the main flexural load case with twelve $250 \mathrm{kN}$ trucks in the center of the main span of the Mentue bridge. The accuracy of the reconstructed deflected shape is very good, with less than $5 \%$ error. By comparison, the results obtained using polynomials are significantly less accurate. The value obtained using only one boundary condition is much larger than the real value, whereas the value using boundary conditions at each end of the bridge is smaller.

Figure 11 shows that, even for comparatively smaller deflections when the adjacent span is loaded, the results remain quite good. For all load cases of the loading test, the deflected shape reconstructed from pre-calculated shapes yielded very good results, even in cases with very small applied loads. 


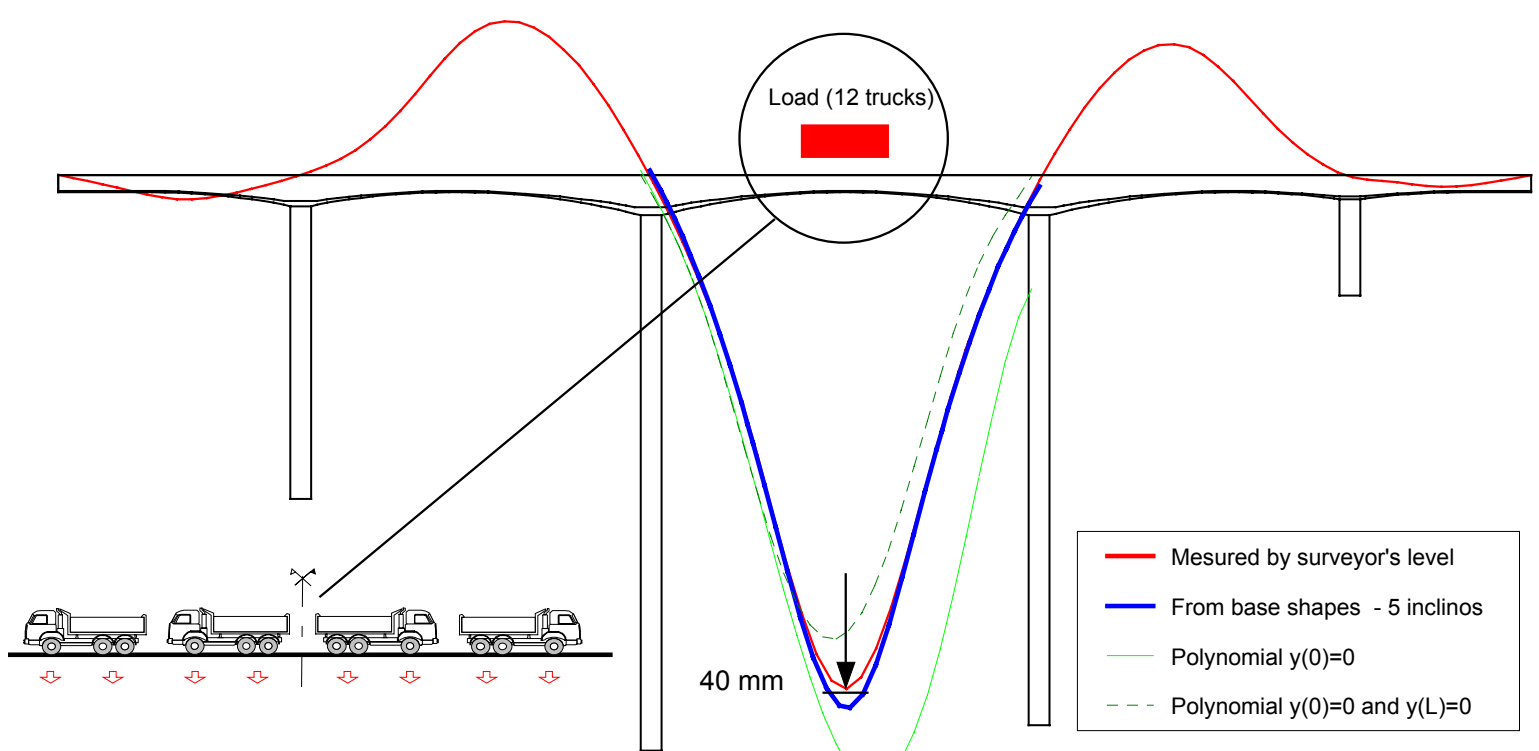

Fig. 10 Deflections under the main load case for the Mentue North bridge

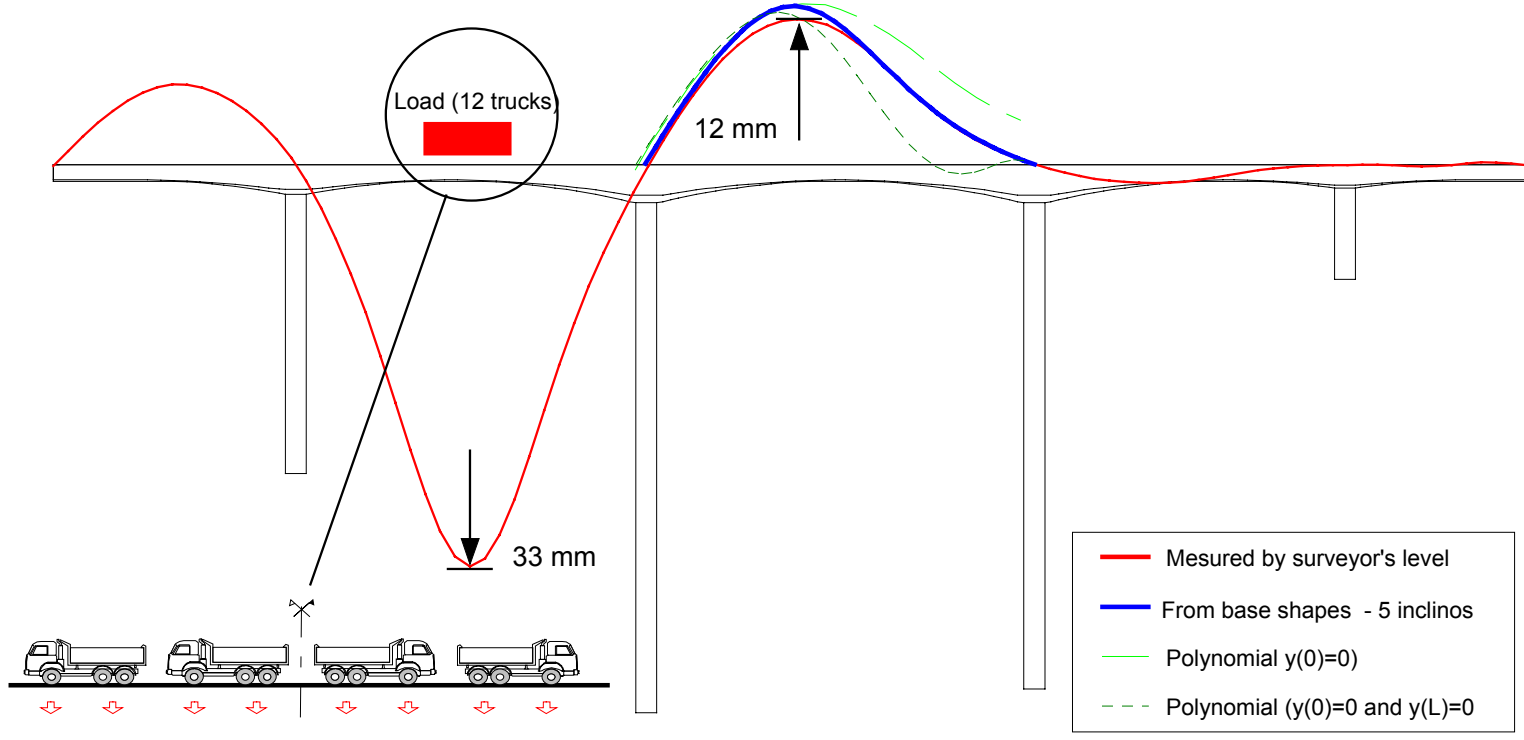

Fig 11 Deflections when the adjacent span is loaded

\section{Conclusions}

An innovative system for the automatic short- and long-term monitoring of the deflections of structures has been presented. Combined with a reconstruction technique based on pre-calculated deflected shapes of the structure, this system has demonstrated its ability to accurately determine the actual deflected shape from the measured rotations. The system has been successfully engaged for the monitoring of a large bridge. The accuracy of the system is demonstrated by comparison with independent methods, in the case of the load test. The first years of experience with the system confirm the value of the system for long-term monitoring.

Combined with other monitoring techniques, the proposed method should open the door to the realization of real intelligent structures. The system is really simple to install, robust, and comparatively inexpensive. Its application to short-term monitoring, may open a new era in the use of regular load testing to control the behavior of bridges.

Further research is under way to determine how to best handle the enormous amount of data recorded on site, and how to also incorporate the temporal information in the evaluation of the bridge condition. 


\section{Acknowledgements}

This research was made possible by a research grant from the Canton de Vaud Highway

Administration. Special thanks go to M. B. Houriet, for allowing us to use his model of the Mentue Bridges. Initial development work on the monitoring with electronic inclinometers was performed with the help of M. Etienne Fest. Finally, we thank the Wyler company in Winterthur, Switzerland, for providing us inclinometers and other supplies at a reduced price.

\section{References}

[1] BURDET O., Automatic Deflection and Temperature Monitoring of a Balanced Canitilever Concrete Bridge, 5th International Conference of Short and medium Span Bridges, Calgary, Canada, 1998.

[2] BURDET O., Load Testing and Monitoring of Swiss Bridges, Comité Européen du Béton, Safety and Performance Concepts, Bulletin d'information n²19, Lausanne, Suisse, 1993.

[3] FAVRE R., BURDET O., CHARIF H., HASSAN M., MARKEY I.F., Enseignements tirés d'essais de charge et d'observations à long terme pour l'évaluation des ponts en béton et le choix de la précontrainte, Office Fédéral des Routes, recherche 83/90, Suisse, 1995.

[4] FAVRE R., CHARIF H., MARKEY I.F., Observation à long terme de la déformation des ponts, Rapport OFR, 86/88, Suisse, 1990.

[5] FEST E., Système de mesure par inclinométrie: développement d'un algorithme de calcul des flèches, DEA Ecole Normale supérieur de Cachan, France, 1997.

[6] PATRON-SOLARES A., GODART B., EYMARD R., Etude des déformations différées du pont de Savines (Hautes-Alpes), Bulletin des Laboratoires des Ponts et Chaussées, 203, 91103, Paris, France, 1996. 